\title{
Local Density of States and Angle-Resolved Photoemission Spectral Function of an Inhomogeneous D-wave Superconductor
}

\author{
Ming Cheng and W. P. Su \\ Department of Physics and Texas Center for Superconductivity, \\ University of Houston, Houston, Texas 77204, USA
}

(Dated: September 26, 2018)

\begin{abstract}
Nanoscale inhomogeneity seems to be a central feature of the d-wave superconductivity in the cuprates. Such a feature can strongly affect the local density of states (LDOS) and the spectral weight functions. Within the Bogoliubov-de Gennes formalism we examine various inhomogeneous configurations of the superconducting order parameter to see which ones better agree with the experimental data. Nanoscale large amplitude oscillations in the order parameter seem to fit the LDOS data for the underdoped cuprates. The one-particle spectral function for a general inhomogeneous configuration exhibits a coherent peak in the nodal direction. In contrast, the spectral function in the antinodal region is easily rendered incoherent by the inhomogeneity. This throws new light on the dichotomy between the nodal and antinodal quasiparticles in the underdoped cuprates.

PACS numbers: 74.20.-z, 74.72.-h, 74.25.Bt
\end{abstract}

\section{INTRODUCTION}

The scanning tunneling microscopy (STM) and the angle-resolved photoemission spectroscopy (ARPES) are two of the most important tools for unraveling the mystery of the high-temperature superconductors (HTS). The existence of a Fermi surface and the d-wave symmetry of the superconducting state are very important properties revealed by ARPES [1] in momentum space. In contrast, STM has provided important complementary information in real space through the measurement of the local density of states (LDOS). A surprising feature of the HTS seen through STM is the conspicuous inhomogeneity [2, 3, 4].

While some of the STM and ARPES data are straightforward to interpret, others are not. The inhomogeneity underlies much of the difficulty as there has not been much theoretical work addressing the effect of inhomogeneous d-wave superconductivity (DSC) on the LDOS 5, 6, 7] and the one-particle spectral function $[8,9,10]$. This paper is intended to partially remedy the situation.

In the absence of a complete theory of HTS, what we have done is to examine various types of inhomogeneity for comparison with the STM and the ARPES data. In this way we hope to extract as much information from the data as possible. In the following, we first introduce the model Hamiltonian and the method of calculation. The calculated results for LDOS and the spectral function are presented and their implications are discussed.

\section{MODEL HAMILTONIAN}

In this work, we focus exclusively on the effect of inhomogeneous d-wave pairing field. We therefore adopt the following Hamiltonian

$$
H=\sum_{k \sigma}\left(\epsilon_{k}-\mu\right) c_{k \sigma}^{\dagger} c_{k \sigma}+\sum_{<i, j>}\left(\Delta_{i, j} c_{i \uparrow} c_{j \downarrow}+H . c .\right)
$$

where $c_{i \sigma}^{\dagger}$ creates an electron on site i with spin $\sigma$, and $c_{k \sigma}^{\dagger}$ an electron with momentum $\mathrm{k}$ and spin $\sigma .\langle i, j\rangle$ is a nearest neighbor pair. The kinetic energy is give by

$\epsilon_{k}=t_{1}\left(\cos k_{x}+\cos k_{y}\right) / 2+t_{2} \cos k_{x} \cos k_{y}+t_{3}\left(\cos 2 k_{x}+\cos 2 k_{y}\right) / 2+t_{4}\left(\cos 2 k_{x} \cos k_{y}+\cos k_{x} \cos 2 k_{y}\right) / 2+t_{5} \cos 2 k_{x} \cos 2 k_{y}$,

where the hopping parameters $t_{1-5}=-0.5951,0.1636$, -0.0519, -0.1117, $0.0510 \mathrm{eV}$ are from $\mathrm{Bi}_{2} \mathrm{Sr}_{2} \mathrm{CaCu}_{2} \mathrm{O}_{8+\delta}$ band structure determined by Norman et al. [11]. $\Delta_{i, j}$ is the $\mathrm{d}$-wave pairing amplitude over a nearest neighbor pair $\langle i, j\rangle$.

For any spatial distribution of $\Delta_{i j}$, the Hamiltonian (1) can in principle be straightforwardly diagonalized after performing a Bogoliubov transformation. In reality, due to the large lattice size involved we need to resort to a special technique for calculating the LDOS.

Gagliano and Balseiro 12 have proposed an efficient method for calculating the resolvent $G_{A}=<\psi_{0} \mid A^{\dagger}(Z-$ 
$H)^{-1} A \mid \psi_{0}>\cdot G_{A}(Z)$ is expressible as a continued fraction [13, 14]

$$
G_{A}(Z)=\frac{<\psi_{0}\left|A^{\dagger} A\right| \psi_{0}>}{Z-a_{0}-\frac{b_{1}^{2}}{Z-a_{1}-\frac{b_{2}^{2}}{Z-\cdots}}} .
$$

where the coefficients $a_{i}$ and $b_{i}$ can be obtained from $A \mid \psi_{0}>$ by repeated application of the Hamiltonian $H$. The Fourier transform of the self-correlation function $C_{A}\left(t-t^{\prime}\right)=<\psi_{0}\left|A^{\dagger}(t) A\left(t^{\prime}\right)\right| \psi_{0}>$ can be recovered from the imaginary part of the resolvent $C_{A}(\omega)=$ $\frac{1}{\pi} \operatorname{Im} G_{A}\left(\omega+i \eta+E_{0}\right)$.

The above method is originally devised for calculating the dynamical properties of quantum many-body systems [12], but it can be easily adapted for our purpose. To calculate the LDOS, we simply take $\left|\psi_{0}\right\rangle$ to be the vacuum and $A$ be $c_{i \sigma}^{\dagger}$. For the spectral function, we choose $c_{k \sigma}^{\dagger}$ instead. The imaginary part of $G_{A}$ then yields the LDOS and the spectral function respectively.

\section{LDOS}

For a homogeneous superconductor, the gap parameter can be directly determined from the measured LDOS. For an inhomogeneous superconductor, it is not trivial to invert the LDOS data for $\Delta_{i j}$. Our approach is to try various $\Delta_{i j}$ configurations to fit the LDOS data. The first set of data we want to fit are the LDOS spectra measured at representative points in underdoped cuprates [15] as shown in Figure 1. Motivated by the experimental gapmap, we consider a cone-shaped distribution [3] of the $\Delta$ order parameter described by the inset of Figure 2. $\Delta$ rises to $4 \Delta_{0}$ at the center of a $400 \times 400$ lattice, but it returns essentially to the background value $\Delta_{0}=0.028 \mathrm{eV}$ for distances larger than ten lattice spacings. The calculated LDOS spectra at a series of points (at a distance 80,8,6,5,4,2,1 from the origin) are displayed in Figure 2. They indeed resemble the measured spectra in Figure 1. In particular, we see that the increase in gapsize as one moves toward the center of the lattice is accompanied by a gradual degradation of the coherence peak. As a reference, the LDOS of a uniform superconductor with $\Delta=4 \Delta_{0}$ is included in Figure 2 .

One implication of the above result is that the largegap incoherent LDOS spectra characteristic of the underdoped cuprates can be interpreted as a rapid rise of the pairing field in a small region, or a nanoscale large amplitude fluctuation. There is no need to invoke a certain unknown charge-ordered zero-temperature pseudogap state [15] which competes with DSC.

The other notable feature of Figure 2 (which agrees with Figure 1) is that the low energy portions of the LDOS spectral are nearly identical suggesting homoge- neous nodal superconductivity coexisting with the inhomogeneous antinodal feature. Such a contrast between nodal and antinodal excitations is also reflected in the ARPES spectra to be discussed in the next section.

Besides the cone-shaped distribution in Figure 2, we have also considered many other $\Delta$ configurations which do not fit the data so well. Figure 3 shows the LDOS spectra corresponding to a mesa-like $\Delta$ configuration described in the inset $\left(\Delta=4 \Delta_{0}\right.$ within a distance of three lattice spacings away from the origin and $\Delta=\Delta_{0}$ elsewhere). Such an extended region of high $\Delta$ leads to a higher energy coherent peak in the LDOS spectra near the center of the mesa. It is quite clear that the data in Figure 1 can discriminate such a configuration from the previous configuration of Figure 2. While the fit to the data in Figure 1 may not be unique, Figure 2 is the best one we have come up with so far.

Although we have considered only a single cone-shaped $\Delta$ configuration, we anticipate that in real cuprates there would be a disordered array of cones. As long as the cones do not overlap strongly, the LDOS of the system should resemble that of a single isolated cone.

The second set of data to fit are the FourierTransformed(FT) LDOS. Due to the constraint of computer time, we again consider only one cone. The calculated FT-LDOS spectra at various energies are displayed in Figure 4 together with the measured one 15]. As emphasized by Dell'Anna et al. [6], previous analyses involving impurity scattering of quasiparticles tend to yield LDOS patterns with extended curve-like features [17, 18, 19, 20] in the high intensity regions in momentum space. This is in contrast to the spot-like intensity patterns seen experimentally. A zeroth order approximate calculation by Dell'Anna et al. shows that a mesoscopically inhomogeneous $\Delta$ distribution indeed yields a central spot. Our result in Figure 4 is exact and gives more details than theirs. In particular, the result exhibits high intensity features along the diagonal as well as horizontal and vertical directions in good agreement with experiment. The checkerboard-like intensity modulations of periodicity about four lattice spacings are also reproduced.

\section{SPECTRAL FUNCTION}

The spatial inhomogeneity revealed by the STM data seems to be at odds with the well-defined Fermi arc. In addition, the nodal quasiparticle peak remains wellresolved even in strongly underdoped cuprates. In contrast, the antinodal quasiparticle peak is well-defined only near optimal composition [1, 21]. This contrast has led to the speculation that the nodal and antinodal excitations have different origins 15. One is associated with DSC which dominates in the optimal and overdoped regions, whereas the other is related to an unknown pseu- 
dogap state which competes with DSC and which dominates in the strongly underdoped samples. Other explanations include coupling of the electrons with the $(\pi, \pi)$ magnetic excitations 22 and a scattering mechanism operating mainly on the antinodal quasiparticles 21].

We have seen in the previous section that the incoherent LDOS spectra in the underdoped samples can be explained in terms of inhomogeneous DSC. Here we attempt to do the same for the ARPES spectra. Figure 5 is the calculated spectral density for a disordered (randomly positioned) array of cones near the nodal direction (5a) and antinodal direction (5b), both momenta are located on the Fermi surface. The nodal quasiparticle peak is indeed well-resolved. The antinodal peaks are broader, but they are still resolved. This is because the $\Delta$ distribution in Figure 5 is dominated by low $\Delta$ values.

To simulate the incoherent antinodal spectral functions seen experimentally in underdoped samples, we fabricate a more disordered $\Delta$ configuration in Figure 6 . The antinodal spectra are indeed incoherent, whereas the nodal one remains coherent. We have examined other $\Delta$ configurations, the contrast between the nodal and antinodal spectral features seems to be generic for inhomogeneous DSC independent of the details of the inhomogeneity. Such a result is actually reasonable because the nodal quasiparticles have vanishingly small excitation energies independent of the magnitude of $\Delta$, therefore they can propagate freely in any inhomogeneous superconductor. For the opposite reason, the energy of an antinodal quasiparticle is very sensitive to $\Delta$, high energy antinodal quasiparticles are confined to regions of high $\Delta$.

\section{DISCUSSION AND CONCLUSION}

For simplicity, we have limited ourselves to only one kind of inhomogeneity in this paper, the inhomogeneous pairing field. In real cuprates, impurities are present as well as short-range antiferromagnetism. They could also affect the LDOS [7, 23] and the spectral weight function. Further theoretical study is required to include those effects.

Despite the limitation, our results so far support the following conclusions: (1) Inhomogeneous DSC is an important determining factor in the LDOS and ARPES spectra; (2) It can explain many unusual features of the experimental spectra without invoking an unknown state which competes with DSC, at least for optimally doped and moderately underdoped systems. The "dichotomy" between nodal and antinodal excitations seems to be a mere consequence of the inhomogeneous d-wave pairing field.

This work was partially supported by the Texas Center for Superconductivity, the Robert A. Welch Foundation (grant number E-1070), and the National Science Council of Taiwan under contract number NSC 92-2112-M-110-
006. We thank Degang Zhang and Hongyi Chen for useful conversation. One of the authors (WPS) thanks S. F. Tsay and the Deparment of Physics at the National Sun YatSen University for their hospitality during summer 2004.

[1] Andrea Damascelli, Zahid Hussain, and Zhi-Xun Shen, Rev. Mod. Phys. 75, 473(2003).

[2] S. H. Pan, J. P. O'Neal, R. L. Badzey, C. Chamon, H. Ding, J. R. Engelbrecht, Z. Wang, H. Eisaki, S. Uchida, A. K. Gupta, K.-W. Ng, E. W. Hudson, K. M. Lang, and J.C. Davis, Nature 413, 282(2001)

[3] C. Howald, P. Fournier, and A. Kapitulnik, Phys. Rev. B 64, 100504(2001).

[4] K. M. Lang, V. Madhavan, J. E. Hoffman, E. W. Hudson, H. Eisaki, S. Uchida, and J. C. Davis, Nature 415, 412(2002)

[5] Z. Wang, Jan R. Engelbrecht, Shancai Wang, Hong Ding, and Shuheng H. Pan, Phys. Rev. B 65, 064509(2002).

[6] L. Dell'Anna, J. Lorenzana, M. Capone, C. Castellani, and M. Grilli, Phys. Rev. B 71, 064518(2005).

[7] W. A. Atkinson, Phys. Rev. B 71, 024516(2005).

[8] I. Martin, G. Ortiz, A. V. Balatsky, and A. R. Bishop, Int. J. Mod. Phys. B 14, 3567(2000).

[9] Degang Zhang, Phys. Rev. B 66, 214515(2002).

[10] D. Podolsky, E. Demler, K. Damle and B. I. Halperin, Phys. Rev. B 67, 094514(2003).

[11] M. R. Norman, M. Randeria, H. Ding, and J. C. Campuzano, Phys. Rev. B 52, 615(1994).

[12] E. R. Gagliano and C. A. Balseiro, Phys. Rev. Lett. 59, 2999(1987).

[13] R. Haydock, V. Heine, and M. J. Kelly, J. Phys. C 5, 2845(1972); 8, 2591(1975).

[14] G. Grosso and G. P. Parravicini, Solid State Physics, p. 20(Academic Press, 2000).

[15] K. McElroy, D.-H. Lee, J. E. Hoffman, K. M. Lang, E. W. Hudson, H. Eisaki, S. Uchida, J. Lee, and J. C. Davis, cond-mat/0404005

[16] S. Sanna, G. Allodi, G. Concas, A. D. Hillier, and R. De Renzi, Phys. Rev. Lett. 93, 207001 (2004).

[17] Q.-H. Wang and D.-H. Lee, Phys. Rev. B 67, 020511(2003).

[18] Degang Zhang and C. S. Ting, Phys. Rev. B 67, 100506(R)(2003).

[19] L. Zhu, W. A. Atkinson, and P. J. Hirschfeld, Phys. Rev. B 69, 060503 (2004).

[20] T. Pereg-Barnea and M. Franz, Phys. Rev. B 68, 180506(2003).

[21] X. J. Zhou, T. Yoshida, D.-H. Lee, W. L. Yang, V. Brouet, F. Zhou, W. X. Ti, J.W. Xiong, Z. X. Zhao, T. Sasagawa, T. Kakeshita, H. Eisaki, S. Uchida, A. Fujimori, Z. Hussain, and Z.-X. Shen, Phys. Rev. Lett. 92, 187001(2004).

[22] Z. X. Shen and J. R. Schriffer, Phys. Rev. Lett. 78, 1771(1997).

[23] C. T. Chen and N. C. Yeh, Phys. Rev. B 68, 220505(2003).

Fig 1: LDOS spectra measured at representative points in underdoped cuprates, data taken from ref. [15].

Fig 2: Calculated LDOS spectra for the spiky $\Delta$ con- 
figuration described in the inset. The curve labeled 'uniform $\Delta^{\prime}$ is included as a reference. It is the LDOS spectrum for a uniform $\Delta=4 \Delta_{0}$.

Fig 3: Calculated LDOS spectra(at a distance $43,4,3,2,1,0$ from the origin) for the $\Delta$ configuration described in the inset.

Fig 4: Calculated FT-LDOS (left column) for a cone-shaped $\Delta$ distribution at various biases $(\omega=$ $-8,-16,-26 \mathrm{meV}$ from top to bottom) compared with data (right column) taken from ref. [15].

Fig 5: Spectral functions along the nodal and antinodal directions corresponding to the $\Delta$ configuration on the left panel.

Fig 6: Same as Figure 5 for a different $\Delta$ distribution. 
This figure "fig1.jpg" is available in "jpg" format from: http://arxiv.org/ps/cond-mat/0508358v1 


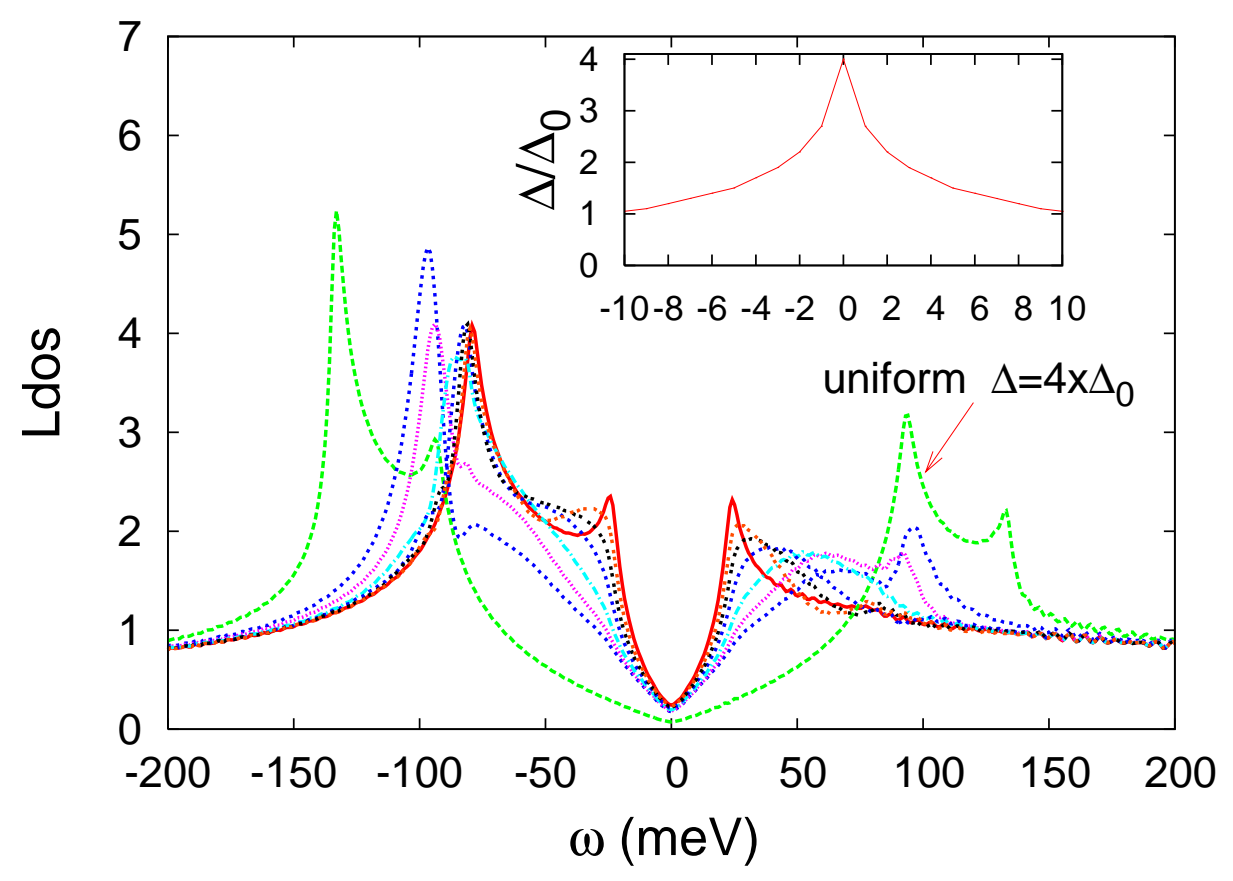




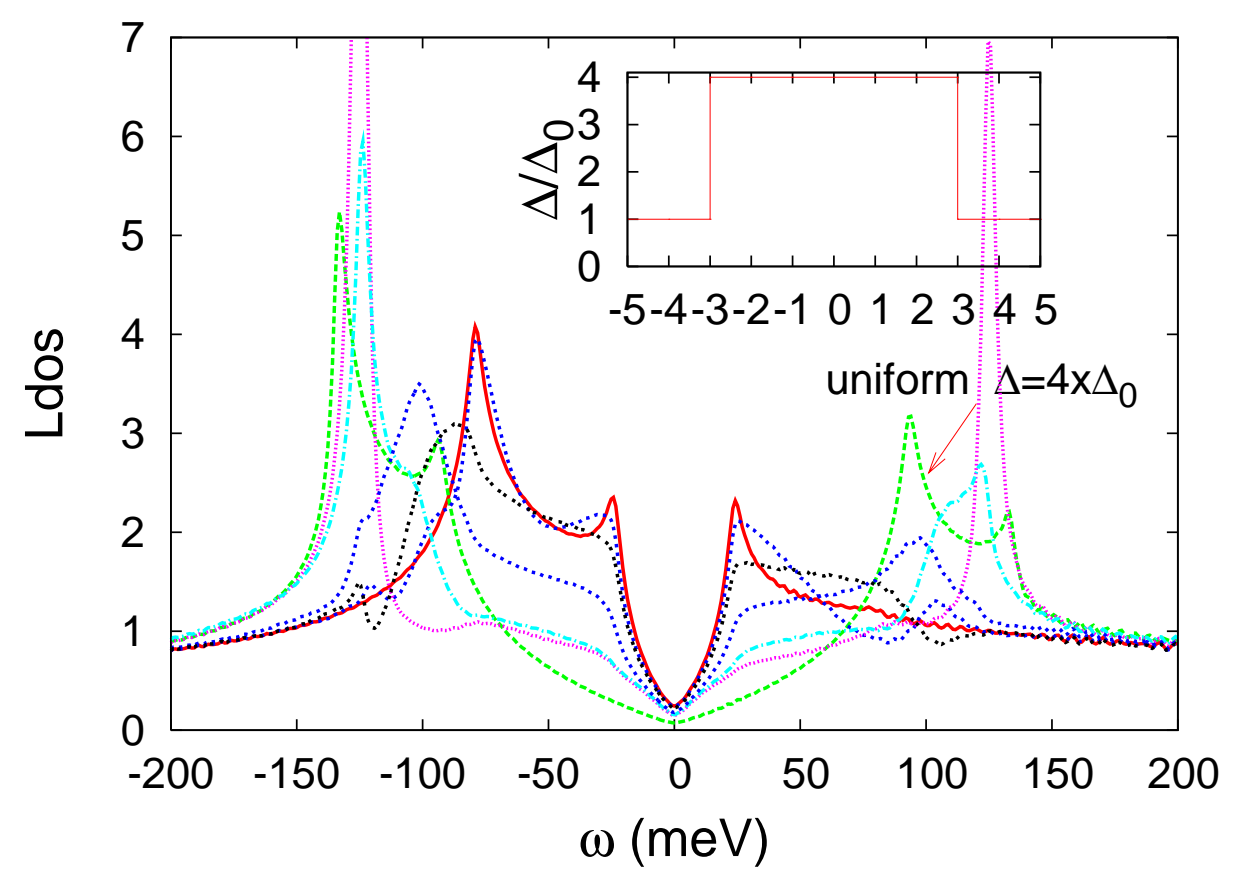


This figure "fig4.jpg" is available in "jpg" format from: http://arxiv.org/ps/cond-mat/0508358v1 


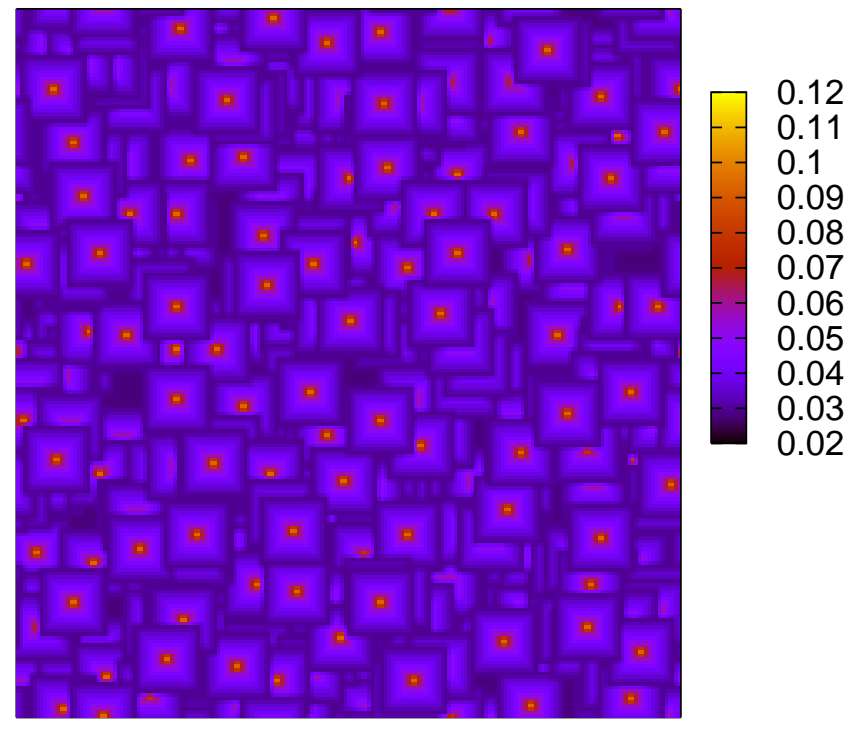




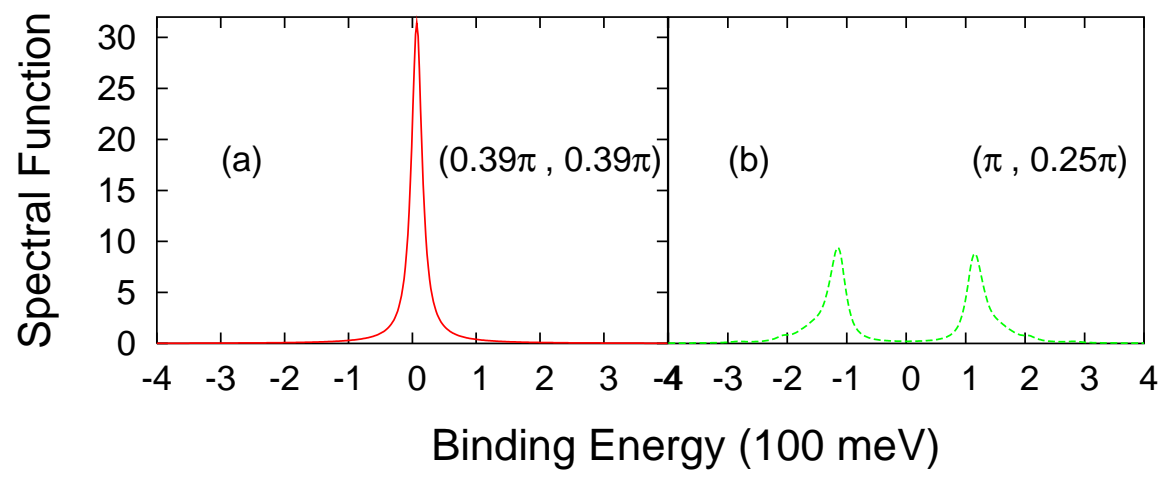


This figure "fig6a.jpg" is available in "jpg" format from: http://arxiv.org/ps/cond-mat/0508358v1 


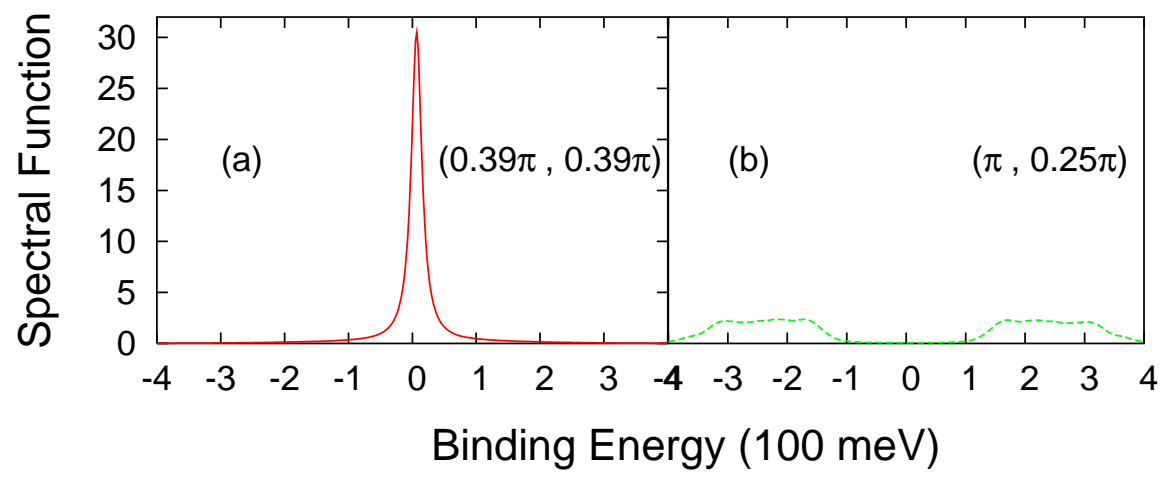

